\title{
A Study of Multi-Signal Monitoring System Establishment for Hemodynamic Energy Detection
}

\author{
YeonSoo Shin ${ }^{1}$, DuckHee Lee ${ }^{1}$ \\ ${ }^{1}$ Korea Artificial Organ Center, \\ College of Medicine, Korea University. \\ Anam-Dong 5ga, Seonbuk-Gu, \\ Seoul 136-701, Korea
}

\author{
SeungJoon Song ${ }^{3}$ \\ ${ }^{3}$ Department of Convergence Biomedical Engineering \\ Daelim University College \\ Imgoklo29 Dongan-gu \\ Anyang-si Gueonggi-do \\ 431-715, Korea
}

\author{
ChiBum Ahn ${ }^{2}$ \\ ${ }^{2}$ Department of Mechanical \& Biomedical Engineering, \\ Engineering of College, Kangwon University \\ Kangwondaehak-gil, Chuncheon-si, \\ Gangwon-do, 200-701, Korea
}

\author{
Kyung Sun ${ }^{1,4}$ \\ ${ }^{4}$ Department of Thoracic and Cardiovascular Surgery, \\ College of Medicine, Korea University \\ Anam-Dong 5ga, Seonbuk-Gu, \\ Seoul 136-701, Korea
}

\begin{abstract}
Deaths due to cardiovascular diseases are increasing worldwide, and multi-signal monitoring systems to diagnose such diseases are under development. However, only a few researches are underway for devices that monitor hemodynamic energy, which is a marker for pulsatile flow generated by the contraction and relaxation of the heart. Therefore, this study aimed to integrate multiple monitoring devices into a single device, while also incorporating hemodynamic energy monitoring. Blood pressure and flow were measured with two channels each, while electrocardiogram (ECG), photoplethysmography (PPG), and temperature were measured with one channel each. All signals were processed at hardware level, and then converted into analog voltage. The seven signals were then converted into digital signals with a data acquisition board (DAQ). The software was developed with Labview $^{\text {TM }}$ (National Instruments, U.S.A) to form a graphic user interface (GUI) on a tablet computer (ACER, U.S.A) through USB 2.0, to allow for monitoring and analysis of the signals obtained. Development of this system successfully formed a multi-signal monitoring system that integrates multiple signals into one device. Future directions include development of cardiovascular diagnosis algorithm and evaluation of the system via preclinical animal experiments.
\end{abstract}

Keywords-Cardiovascular disease; hemodynamic energy; monitoring system; Electrocardiogram; Photoplethysmography; Pressure; Flow

\section{INTRODUCTION}

According to the World Health Organization (WHO), the leading cause of death worldwide in 2011 was cardiovascular disease, which was responsible for 1.7 million deaths. WHO also predicted that two million people are expected to die from the same cause in 2015. Devices for diagnosing cardiovascular disease are under active development worldwide, and are differentiated into several types of sensors and devices. The heart maintains a pulsatile flow by its contractions and relaxations [1-3], and multiple researchers have analyzed the unique properties of pulsatile flow and compared them with those of nonpulsatile flow [4-11]. Multiple advantages of pulsatile flow during cardiovascular bypass have been reported, such as its similarities to the natural beating of the heart, as well as keeping the blood vessels open [12]. Also, a study utilizing the concept of hemodynamic energy characteristic of pulsatile flow suggests that pulsatile flow has greater hemodynamic energy, and has advantage in perfusion [13]. In 2002, Nitzan proposed a signal-processing algorithm that calculates the pulse transit time (PTT) from the R-wave and the photoplethysmography (PPG) recorded from the earlobes, fingers, and toes. The study reported the correlation between PTT and blood pressure in average adults [14]. Development of a system that integrates the conventional biological signals and hemodynamic energy will result in a system that can be used for both diagnosis of diseases as well as efficacies of treatments. However, such integrated system is currently difficult to find. Therefore, this study aimed to develop a multisignal monitoring system that integrates the conventional signals including ECG, PPG, and temperature, as well as hemodynamic energy as Overall System.

\section{OVERALL SYSTEM CONFIGURATION}

The system has a total of seven channels. Pressure and flow are measured in two channels each, while ECG, PPG, and temperature are measured with one channel each. Figure 1 shows the overall configuration of the system, where multiple signals are relayed to a single input, processed by analog-todigital converting (ADC), which then can be saved and analyzed. 


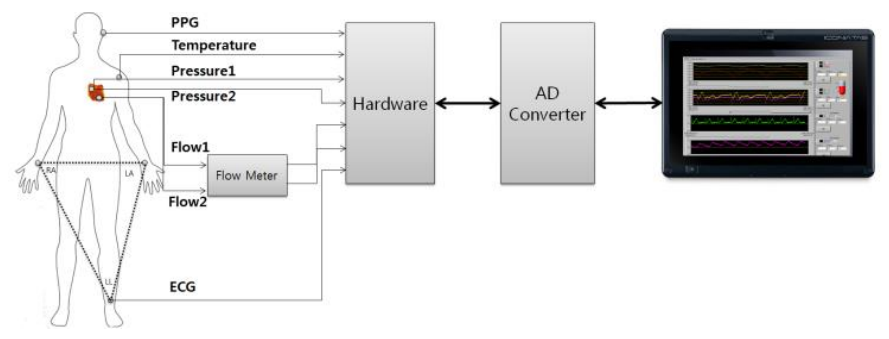

Fig. 1. System Blockdiagram

\section{HARDWARE SYSTEM STRUCTURE}

Pressure is measured by deflection method with the ST AM100 amplifier (Senstech, Korea), a dynamic strain amplifier operating on AC $220 \mathrm{~V}$. A low pass filter at $10 \mathrm{~Hz}$ was used, and an internal filter was used to amplify the gain by 200 times. The PS9030VY pressure sensor was used (Sensortechnics, Germany). Output is shown in voltage between $0 \sim 10 \mathrm{~V}$, and can measure 0 4000 ustrain. Flow was measured with the TS410 flow meter (Transonic System, U.S.A), a tubing flow meter operating on AC $220 \mathrm{~V}$. Flow detected with the ME11PXL probe (Transonic System, U.S.A) was expressed in voltage between $0 \sim 1 \mathrm{~V}$. Pulses were measured with EP520 (Laxtha, Korea), a photosensor that can be attached to the earlobes. The applied voltage of the EP520 was $5 \mathrm{~V}$, and the LED Driving current control voltage was $0.38 \mathrm{~V}$. The LED Driving voltage required approximately three seconds of response time before showing stable waveforms. To measure temperature, K-type thermocouple sensors were used with a Ktype thermocouple amplifier chip, AD595 (Analog Device, Inc, U.S.A), which operates on $5 \mathrm{~V}$. Change in temperature was detected as a voltage change of $10 \mathrm{mV}$ per temperature change of $1{ }^{\circ} \mathrm{C}$. ECG was measured with the Einthoven's method with standard leads I, II, and III. Snap electrodes (Laxtha, Korea) were used in conjunction with AD620 amplifier (Analog Device Inc, U.S.A) operating on 5 V. ECG signals were differentially amplified, and noise was eliminated with bandpass filtering and $60 \mathrm{~Hz}$ notch filtering by LM324 amplifier (Analog Device, Inc, U.S.A). The developed ECG hardware was processed into printed circuit boards (PCB), using Pads 9.4 software (Mentor Graphics, U.S.A). Because ECG, temperature, and pulse signals all operate on $5 \mathrm{~V}$, the power input was supplied from an ADC board, NI USB-6009 (National Instruments, U.S.A). Because all signals are collected in analog form, they must be converted into digital signals before being processed by a computer. Therefore, NI USB-6009, which has 14 bit resolution, sample rate of $48 \mathrm{kS} / \mathrm{s}$ and accuracy range of $1.53 \mathrm{mV}$ was used to convert the signals from analog to digital. These signals were transferred to a tablet PC (ACER, U.S.A) via USB 2.0 connection and measured using the LabView ${ }^{\mathrm{TM}}$ software.

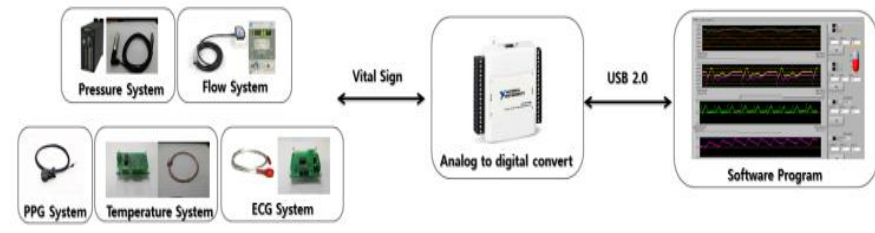

Fig. 2. Integrated System Configuration

\section{CONCEPT INTRODUCTION OF HEMODYNAMIC ENERGY}

Shepard's equation, which calculates the hemodynamic energy in a pulsatile flow using pressure and flow rate was applied in this system [15].

$$
\operatorname{EEP}(\mathrm{mm} \mathrm{Hg})=\left(\int f p d t\right) /\left(\int f d t\right)
$$

Energy equivalent pressure (EEP) is the amount of energy required to move a unit volume of blood across a unit distance, expressed in pressure units. f represents the flow rate $(\mathrm{L} / \mathrm{min})$, while $p$ represents arterial pressure $(\mathrm{mm} \mathrm{Hg})$. EEP is determined by first calculating hemodynamic power curve, which is an integration of the product of flow rate and pressure during one cardiac cycle, and then dividing it by the pump flow-rate curve, which is an integration of the flow rate over time.

$\operatorname{SHE}\left(\mathrm{ergs} / \mathrm{cm}^{3}\right)=1,332\left[\left(\left(\int f p d t\right) /(f d t)\right)-M A P\right]$

Surplus hemodynamic energy (SHE) is the excess energy after subtracting the amount necessary for basic circulation [16]. Therefore, SHE is expected to be 0 in nonpulsatile flows where EEP equals mean arterial pressure (MAP), but positive in pulsatile flows where EEP is greater than MAP. Based on the hemodynamic energy equations, software was developed with the Labview ${ }^{\mathrm{TM}}$ software to monitor hemodynamic energy EEP and SHE in pulsatile flow generated by a Korean pulsatile biventricular pump, TPLS (Twin Pulse Life Support) [17].

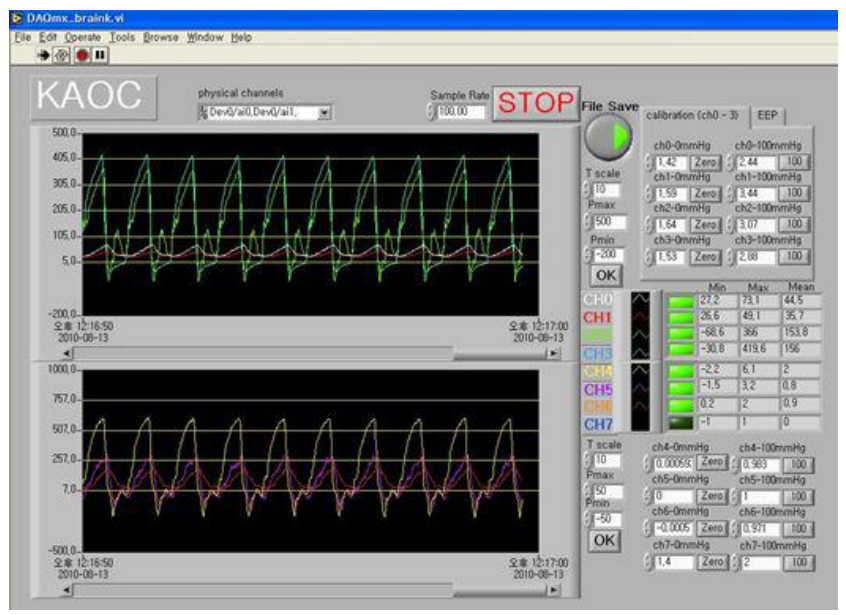

Fig. 3. Measurement of Hemodynamic energy

\section{SOFTWARE FOR VITAL Sign MEASUREMENT}

One cycle of ECG is comprised of $\mathrm{P}, \mathrm{Q}, \mathrm{R}, \mathrm{S}$, and T-wave, out of which the QRS complex occurs during ventricular polarization and typically reside in higher frequency area. The $\mathrm{P}$-wave is caused by atrial contractions, while the T-wave is caused by ventricular contractions, and both typically reside in the lower frequencies. The QRS complex plays a fundamental and critical role in ECG analysis; once the analysis of the QRS complex is complete, ST waves, PR waves, and RR intervals can also be analyzed. Therefore, ECG waveforms were displayed using secondary band-pass filters, $60 \mathrm{~Hz}$ notch filters, and FIR filtering at a software level to distinguish the QRS complex. 
The pulse rate signal may be affected by the patient's respiration or skin condition, which affects the current and amplitude of the signals. Therefore, the patient must remain stationary to obtain stable waveforms. A baseline equation can be applied to calculate the pulse rate by comparing the occurrences of peak values.

$$
\text { Pulse Rate }=\frac{60(\mathrm{sec})}{\text { Duration Between Peaks }}
$$

Because the AD595 chip displays $1{ }^{\circ} \mathrm{C}$ of temperature change per $10 \mathrm{mV}$ of voltage change, equation 4 was applied to the software to show the temperature.

$$
\text { Temperature }=\text { Measured voltage }(\mathrm{mV}) \times 100
$$

As shown in figure 4, ECG and pulse signals were graphed in Labview ${ }^{\mathrm{TM}}$ in real time, and temperature was displayed by the thermometer icon.

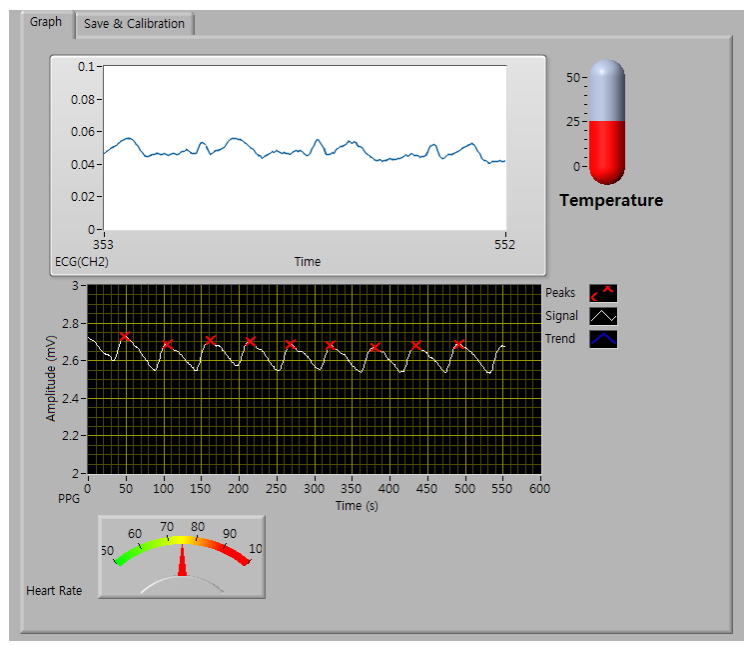

Fig. 4. Measurement of Complex Vital Signal

\section{RESUlT OF INTEGRATED HARDWARE AND SOFTWARE}

Hardware and software for multiple vital signals were integrated into a device and simplified. All seven analog signals can be converted into digital signals and transferred to the DAQ board and a portable tablet computer via USB 2.0, which can then be analyzed with the Labview ${ }^{\mathrm{TM}}$ software, as seen in figure 5. By combining multiple softwares, changes in individual signals can be graphed in real time onto a single screen. Sampling rate and duration can be adjusted as needed, and the raw data can be saved in text or excel format, which can then be analyzed statistically.

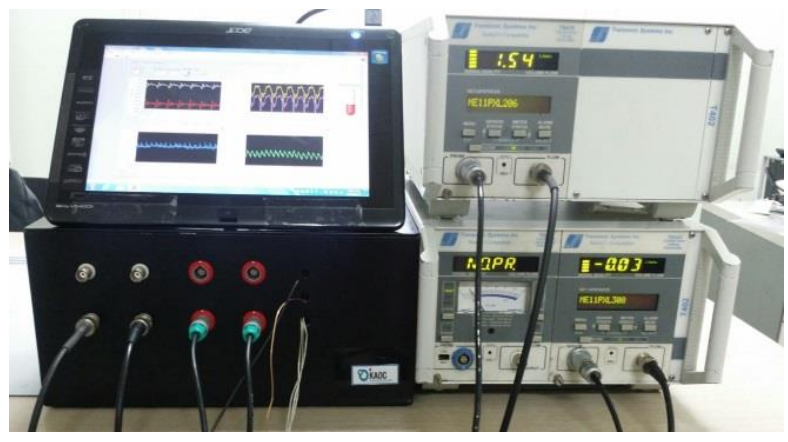

Fig. 5. Result of Integrated System

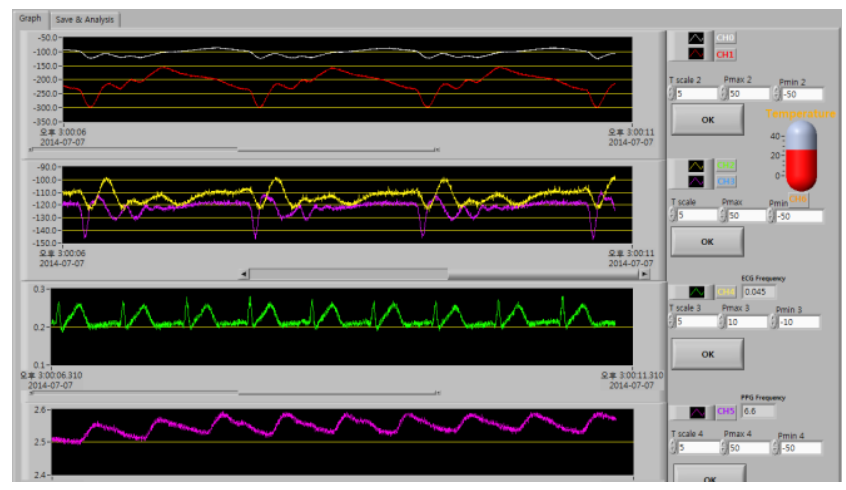

Fig. 6. Software User Interface

\section{CONCLUSION}

In this study, a system was developed to combine measurements of signals necessary for calculation of hemodynamic energy, as well as other monitoring parameters, including pressure, flow, ECG, PPG, and temperature, for the purpose of the development of an integrated multi-signal monitoring system. Although the analog measurement of temperature change showed high precision at $10 \mathrm{mV}=1{ }^{\circ} \mathrm{C}$, the converted digital value showed lower precision in comparison. This can be attributed to the noise from the power input, generated upon connection to the USB-6009 DAQ board. Efforts are being made to reduce the effect of this noise on measurement precision. Future directions include replacing cable connection with wireless connection in order to simplify the structure of the system. Furthermore, hemodynamic energy markers will be integrated with ECG and PPG to develop a vascular monitoring system. Once performance and safety of this newly developed system prove to be suitable for clinical use after in vitro and in vivo experiments, it may be possible to utilize this system in both monitoring and diagnosis for circulatory conditions.

\section{ACKNOWLEDGMENT}

This research was funded by the Ministry of Health \& Welfare, Republic of Korea (grant number: A121157)

\section{REFERENCES}

[1] Marroudis C, "To pulse or not to pulse", Ann Thorac Surg 1978. 25: 259-71.

[2] Hichey PR, Buchley MJ, Philbin DM, "Pulsatile and nonpulsatile cardiopulmonary bypass review of a counterproductive controversy", Ann thorac Surg 1983. 36: 720-37

[3] Hornick P, Taylor K, "Pulsatile and nonpulsatile perfusion: the continuing controversy", J Cardiothorac Vasc Anesth 1997. 11: 310-5

[4] Drakos SG, Charitos CE, Ntalianis A, et al, "Comparison of pulsatile with nonpulsatile mechanical support in a porcine model of profound cardiogenic shock", ASAIO J 2005. 51: 26-9.

[5] Undar A, Rosenberg G, Myers JL, "Major factors in the controversy of pulsatile versus nonpulsatile flow during acute and chronic cardiac support”, ASAIO J 2005. 51: 173-5.

[6] Brandes H, Albes JM, Conzelmann A, Wehrman M, Ziemer G, "Comparison of pulsatile and nonpulsatile perfusion of the lung in an extracorporeal large animal model”, Eur Surg Res 2002. 34:321-9

[7] Undar A, Masai T, Frazier OH, Fraser CD. Pulsatile and nonpulsatile flow can be quantified in terms of energy equivalent pressure during cardiopulmonary bypass for direct comparisons, ASAIO J 1999. 610-4.

[8] Lodge AJ, Undar A, Daggett CW, Runge Tm, Calhoon JH, Ungerleiger $\mathrm{RM}$, "Regional blood flow during cardiopulmonary bypass and after 
circulatory arrest in an infant model", Ann Thorac Surg 1997. 63:124350 .

[9] Ciadullo Rc, Schaff HV, Flaherty JT, Donahoo JS, Gott VL, "Comparison of regional myocardial blood flow and metabolism distal to critical coronary stenosis in the fibrillating heart during alternate periods of pulsatile and nonpulsatile perfusion", J Thorac Cardiovasc Surg 1978. 75:193-205.

[10] Maddoux G, Pappas G, Jenkins M, et al. "Effect of pulsatile and nonpulsatile flow during cardiopulmonary bypass on left ventricular ejection fraction early after aortocoronary bypass surgery", Am J Cardiol 1976. 37:1000-6.

[11] Murkin JM, Marzke JS, Buchan AM, Bentley C, Wong CJ. "A randomized study of the Influence of perfusion technique and $\mathrm{PH}$ management strategy in 316 patients undergoing coronary artery bypass Surgery", Mortality and cardiovascular mobidity. J Thhorac Cardiovasc Surg 1955. 110: 340-8.

[12] Shepard RB, Kirklin JW, "Relation of pulsatile flow to oxygen consumption and other variables during CPB", J Thorac Cardiovasc Surg 1969. 58: 694-702.

[13] Ji B, Undar A: "An evaluation of the benefits of pulsatile versus nonpulsatile perfusion during cardiopulmonary bypass procedures in pediatric and adult cardiac patients", ASAIO J 2006. 52: 357-61.

[14] M. Nitzan, B. Khanokhm and Y. Slovik, "The difference in pulse trasit time to the toe and finger measured by photoplethysmography", Physial. Meas, 2002. 23:85-93,

[15] Shepard RB, Simpson DC, Sharp JF, "Energy Equivalent Pressure", Arch Surg 1966. 96:93: 790-40

[16] Undar A, Zapanta CM, Reibson JD, et al. "Precise quantification of pressure flow waveforms of a pulsatile ventricular assist device", ASAIO J. 2005. 51(1): 56-9.

[17] Jung Joo Lee, Choon Hak Lim, Ho Sung Son, et al. "In vitro evaluation of the performance of Korean pulsatile ECLS(T-PLS) using precise quantification of pressure- flow waveforms", ASAIO J. 2005. 51(5): 604-8. 